\title{
Epidemiological Characteristics of Norovirus Associated with Sporadic Gastroenteritis among Children from the 2006/2007 to 2011/2012 Season in Nara Prefecture, Japan
}

\author{
Masaki Yoneda Akiko Okayama Yoshiteru Kitahori \\ Virology and Epidemiology Division, Nara Prefecture Institute of Health, Sakurai City, Japan
}

\section{Key Words}

Norovirus · Sporadic gastroenteritis · Children

\begin{abstract}
The present study aimed to describe the epidemiological characteristics of norovirus (NoV) associated with sporadic gastroenteritis in regional populations of Nara Prefecture, Japan, from the $2006 / 2007$ to $2011 / 2012$ epidemic season. Fecal specimens of sporadic gastroenteritis collected between September 2006 and August 2012 in Nara Prefecture were examined for the presence of NoV by reverse transcription-polymerase chain reaction. The NoV genotype was determined by nucleotide sequence analysis. In total, $274 \mathrm{NoVs}$ associated with sporadic gastroenteritis were identified. We detected 10 different NoV genotypes: Gl/3, Gl/4, Gl/8, GII/2, GII/3, GII/4, GII/6, GII/7, GII/12, and GII/13. A high NoV detection rate of $35.9 \%$ was identified in 1 -year-old children. Among the 274 NoV isolates, 142 were obtained from males and 131 were obtained from females (the source of one was unknown). The most prevalent genotype was Gll/4, accounting for 117 of the 192 different NoVs identified by sequencing. More epidemiological data will be required to determine the epidemiological characteristics of NoVs in other areas of Japan.

Copyright $\odot 2013$ S. Karger AG, Basel
\end{abstract}

Noroviruses (NoVs) (formerly called Norwalk viruses) are a major cause of acute gastroenteritis in children and adults worldwide. Most children have at least one NoV infection within the first 5 years of life [1]. NoVs are nonenveloped viruses with a single-stranded, positivesense, polyadenylated RNA genome and belong to the family Caliciviridae along with four other genera (Vesivirus, Lagovirus, Sapovirus, and Nevovirus). Studies investigating neutralizing antibodies against NoV have not been possible because of the absence of regular tissue culture systems, however recent advances in NoV sequencing have enabled their genomic characterization. The genome of NoV encodes three open reading frames (ORFs): ORF1, ORF2, and ORF3 [2, 3]. ORF1 encodes nonstructural proteins, ORF2 encodes the major capsid protein, and ORF3 encodes a minor structural protein $[2,3]$. The majority of human NoVs are classified into two genogroups, GI and GII, which are further subdivided into more than 30 genotypes on the basis of their capsid and/ or polymerase genes [4]. Despite this heterogeneity, 1 genotype, GII/4, currently causes the majority of infections worldwide [5]. Four large pandemics (1995-1996, 2002-2003, 2004-2005, and 2006-2007) have been identified, each corresponding to the emergence of one or two new GII/4 variants [6]. As seen in many countries, the

\section{KARGER}

E-Mail karger@karger.com

www.karger.com/int
(C) 2013 S. Karger AG, Basel

0300-5526/14/0571-0031\$39.50/0
Masaki Yoneda

Virology and Epidemiology Division

Nara Prefecture Institute of Health, 1000 Odono

Sakurai City, Nara 633-0062 (Japan)

E-Mail yoneda-masaki@office.pref.nara.lg.jp 
GII/4 variant 2006b became epidemic during the 2006/2007 epidemic season in Japan [7]. Therefore, understanding the molecular epidemiology of GII/4 is very important.

Since the 2006/2007 season, molecular surveillance of NoV infections has been conducted in Nara Prefecture, Japan $[8,9]$. In this study, we describe the epidemiological characteristics of NoV associated with sporadic gastroenteritis in regional populations in Nara Prefecture from the $2006 / 2007$ to $2011 / 2012$ epidemic season. Between September 2006 and August 2012, 1,159 stool samples were collected from patients with acute nonbacterial sporadic gastroenteritis from 13 hospitals in Nara Prefecture. All the patients were $<15$ years old. This study included only fecal specimens from sporadic cases, excluding outbreaks, as assessed by the pediatrician's interview of the patient. For the 1,159 stool specimens, NoVs $(\mathrm{n}=274)$, rotaviruses $(\mathrm{n}=256)$, adenoviruses $(\mathrm{n}=37)$, and other viruses $(\mathrm{n}=51)$ were detected. Of the $274 \mathrm{NoV}$ strains, $75,47,40,45,33$, and 33 strains were obtained during the 2006/2007, 2007/2008, 2008/2009, 2009/2010, 2010/2011, and 2011/2012 epidemic seasons, respectively. In this study, the annual observation period for NoV gastroenteritis began in September and ended in August of the following year.

The fecal specimens were diluted in Hank's balanced salt solution to obtain $10 \%$ (weight/volume) suspensions and clarified by centrifugation at $1,500 \mathrm{~g}$ for 15 min. The aqueous phase was transferred to a new tube for further purification. Chloroform was added, and the suspension was mixed for $15 \mathrm{~s}$ before centrifugation at $1,500 \mathrm{~g}$ for $15 \mathrm{~min}$. The upper aqueous phase was used for viral RNA extraction. Viral RNA was extracted from the aqueous phase using the QIAmp Viral RNA Mini Kit (Qiagen GmbH, Hilden, Germany) according to the manufacturer's instructions. The extracted viral RNA was dissolved in nuclease-free water and stored at $-80^{\circ}$ until use for reverse transcription-polymerase chain reaction (RT-PCR). RT-PCR was performed using the PrimeScript One-Step RT-PCR Kit Version 2 (Takara, Shiga, Japan) according to the manufacturer's instructions. Reactions were performed under the following conditions: initial hold at $50^{\circ}$ for $30 \mathrm{~min}$ and $94^{\circ}$ for $2 \mathrm{~min}$, followed by 40 cycles at $94^{\circ}$ for $30 \mathrm{~s}, 60^{\circ}$ for $30 \mathrm{~s}$, and $72^{\circ}$ for $30 \mathrm{~s}$. The partial $\mathrm{N}$-terminal capsid region was amplified using the primer pair COG1F [10] and G1SKR [11] for GI viruses or COG2F [10] and G2SKR [11] for GII viruses. When the amplification was insufficient for sequencing, semi-nested PCR was performed using the primer pair G1SKF [11] and G1SKR for GI viruses or G2SKF [11] and G2SKR for GII viruses. To identify NoV genotypes, direct sequencing was performed. PCR amplicons from NoV-positive specimens were separated by $1.5 \%$ agarose gel electrophoresis and purified using the NucleoSpin Extract II Kit (Takara). The purified amplicons were used as templates for direct sequencing. Sequencing of the purified amplicons was performed using the BigDye Terminator Cycle Sequencing Kit and Genetic Analyzer 310 System (Applied Biosystems, Foster City, Calif., USA). Sequencing reactions were performed by an initial denaturation step at $96^{\circ}$ for $1 \mathrm{~min}$, followed by 25 cycles of $96^{\circ}$ for $10 \mathrm{~s}, 50^{\circ}$ for $5 \mathrm{~s}$, and $60^{\circ}$ for $4 \mathrm{~min}$. Nucleotide sequences were aligned using ClustalX, and the distances were calculated by Kimura's two-parameter method. Phylogenetic trees with bootstrap analysis from 1,000 replications were generated by the neighbor-joining method as described previously [4]. Genotype numbers in this study were identified according to Kageyama et al. [4]. GII/4 variants were obtained by phylogenetic clustering with 11 reference strains (see fig. 1). The NoV candidate sequences were deposited in the DDBJ database (http:// www.ddbj.nig.ac.jp) with the following accession numbers: AB751625 to AB751661.

Previously, we reported the results of an epidemiological study of NoVs in Nara Prefecture between April 2006 and March $2008[8,9]$. The present study extended these findings by investigating the difference in the epidemic patterns until August 2012. In total, 192 of the 274 confirmed $\mathrm{NoV}$-positive specimens were genotyped. Of these 192 strains, 6 (3.1\%) belonged to the GI genogroup and 186 (96.9\%) belonged to the GII genogroup. In phylogenetic analyses based on the partial $\mathrm{N}$-terminal capsid region with the reference strains, 10 different NoV genotypes were observed. The GI and GII genogroups were further divided into 3 and 7 genotypes, respectively. In each season, 2-6 genotypes were observed. Only GII/4 was observed in every season. Among the 186 GII strains, 117 strains were classified as GII/4 (62.9\%), with GII/2 (14.0\%), GII/3 (11.8\%), GII/13 (6.5\%), and GII/6 (3.2\%) accounting for fewer strains. NoV GII/7 and GII/12 were detected in less than $2 \%$ of the strains (table 1 ). The high occurrence of the GII/4 genotype was reported in a recent surveillance of NoV epidemics in other areas of Japan [12] as well as in other countries [13]. The NoV detection rate was $35.9 \%$ in 1 -year-old children between the 2006/2007 and 2011/2012 season. Of the 274 obtained strains, 142 were isolated from males and 131 were isolated from females (the source of 1 strain was unknown), resulting in a male-to-female ratio of approximately $1.08: 1$. In this 


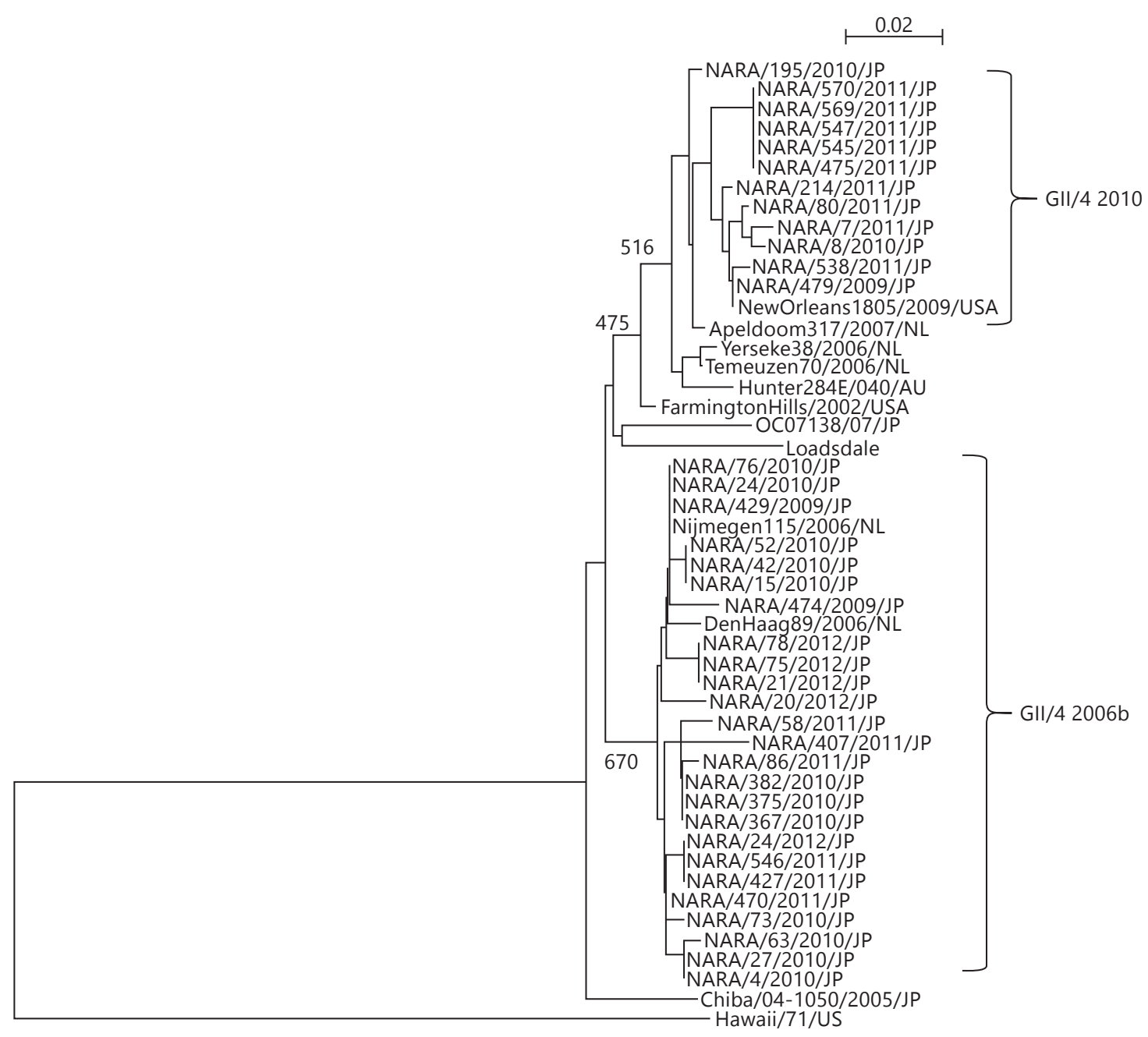

Fig. 1. Phylogenetic analysis based on a 282-bp sequence of the capsid region of $37 \mathrm{GII} / 4$ sequences detected from the 2009/2010 to 2011/2012 season. The numbers on the branches are the bootstrap values for the clusters. The GenBank accession numbers for reference strains used in this analysis are as follows: Hawaii/71/US, U07611; Loadsdale, X86557; FarmingtonHills/2002/USA, AY502023;
Chiba/04-1050/2005/JP, AB220921; Hunter284E/04O/AU, DQ078814; Terneuzen70/2006/NL, EF126964; Yerseke38/2006/NL, EF126963; Nijmegen115/2006/NL, EF126966; DenHaag89/2006/ NL, EF126965; OC07138/07/JP, AB434770; Apeldoorn317/2007/ NL, AB445395, and NewOrleans1805/2009/USA, GU445325. study, $117 \mathrm{GII} / 4$ strains were analyzed. As a result, four GII/4 variants were observed in Nara Prefecture from the $2006 / 2007$ to $2011 / 2012$ season. In addition to the pandemic variant GII/4 2006b, GII/4 sequences clustered with the newly reported variant GII/4 2010 after the 2009/2010 season (fig. 1). Only GII/4 2006b was observed in every season.

NoV has been the etiological agent of many sporadic cases and outbreaks of gastroenteritis in Japan $[4,12,14$,
15]. Despite the limited number of positive samples used to determine seasonality, our results illustrated the great diversity of NoV strains in a limited area of Japan because GI/3, GI/4, GI/8, GII/2, GII/3, GII/4, GII/6, GII/7, GII/12, and GII/13 strains were detected. During the 2008/2009 season, GII/6 emerged as the second most common genotype in different regions of Japan $[12,16]$, and during the $2009 / 2010$ season, a significant increase in the number of GII/2 strains was observed in 
Table 1. NoV genogroups and genotypes isolated in Nara Prefecture from the $2006 / 2007$ to 2011/2012 season

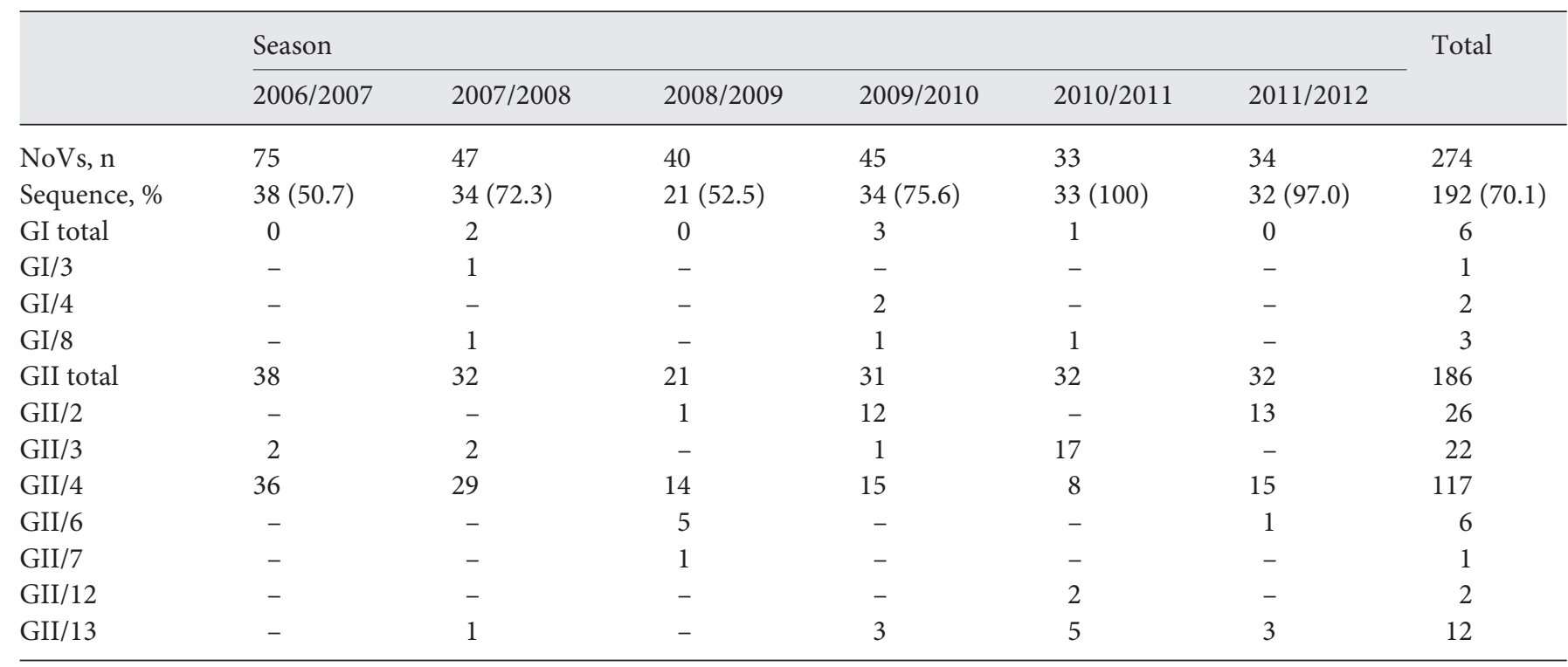

Osaka City [17]. Our results indicated that a similar pattern was observed in sporadic cases in Nara Prefecture in both the seasons. Regional studies are necessary to elucidate the distribution of NoVs in Japan. In a recent study in Finland, $49 \%$ of infants were infected with NoV by the age of 2 years [18]. Although we could not confirm cases of primary infection in this study, 119 of the 192 sequenced strains (62.0\%) were detected in children $\leq 2$ years of age. Thus, the multitude of young viruspositive patients suggested the possibility of primary infection in this study. A recent report suggested that GII/4 caused more severe disease than other NoV genotypes in cases of acute gastroenteritis in young children associated with primary infection [19]. In this study, 88 of the 119 sequenced strains (73.9\%) were GII/4 strains in children $\leq 2$ years of age; therefore, it is necessary to provide appropriate care for severe symptoms. The merit of research in children is that episodes can be examined without the effect of preexisting immunity. Thus, further epidemiological investigations are required to clarify the differences between NoV infections in children. GII/4 is the cause of sporadic cases of pediatric gastroenteritis worldwide $[5,20]$. In this study, GII/4 was the most prevalent genotype detected and was identified in 117 of the 192 sequenced strains. The high prevalence of GII/4 (62.9\%) is consistent with recent epidemiological studies in other areas of Japan and in other countries $[5,12]$. Although our study focused only on the capsid protein, our results revealed the diversity of GII/4 variants in Nara Prefecture. In Belgium, GII/4 2010 variants were identified in December 2009 [21]. More epidemiological data will be required to determine the implication of this variant in other areas of Japan.

Finally, this study analyzed the epidemiological characteristics of $\mathrm{NoV}$ in association with sporadic cases among children in Nara Prefecture and revealed the genetic diversity and age distribution of infection in a local area of Japan. Further studies are required to monitor whether GII/4 2010 variants will change in future. In Japan, a few local institutions are conducting detailed genetic analyses of NoVs. Accumulation of such data in local institutions is important for knowing the distribution of the new GII/4 variant not only in Japan but also in other parts of the world.

\section{Acknowledgement}

The collection of specimens was strongly supported by participants in the Infection Disease Surveillance in Nara Prefecture. We would like to express our deep thanks to them.

\section{Disclosure Statement}

The authors have no conflicts of interest to disclose. 


\section{References}

1 Lopman B, Armstrong B, Atchison C, Gray JJ: Host, weather and virological factors drive norovirus epidemiology: time-series analysis of laboratory surveillance data in England and Wales. PLoS One 2009;4:e6671.

-2 Bull RA, Hansman GS, Clancy LE, Tanaka MM, Rawlinson WD, White PA: Norovirus recombination in ORF1/ORF2 overlap. Emerg Infect Dis 2005;11:1079-1085.

-3 Tsugawa T, Numata-Kinoshita K, Honma S, Nakata S, Tatsumi M, Sakai Y, Natori K, Takeda N, Kobayashi S, Tsutsumi H: Virological, serological, and clinical features of an outbreak of acute gastroenteritis due to recombinant genogroup II norovirus in an infant home. J Clin Microbiol 2006;44:177-182.

4 Kageyama T, Shinohara M, Uchida K, Fukushi S, Hoshino FB, Kojima S, Takai R, Oka T, Takeda N, Katayama K: Coexistence of multiple genotypes, including newly identified genotypes, in outbreaks of gastroenteritis due to Norovirus in Japan. J Clin Microbiol 2004; 42:2988-2995.

5 Bull RA, Tu ET, McIver CJ, Rawlinson WD, White PA: Emergence of a new norovirus genotype II.4 variant associated with global outbreaks of gastroenteritis. J Clin Microbiol 2006;44:327-333.

-6 Siebenga JJ, Vennema H, Renckens B, de Bruin E, van der Veer B, Siezen RJ, Koopmans M: Epochal evolution of GGII.4 norovirus capsid proteins from 1995 to 2006 . J Virol 2007;81: 9932-9941.

$>7$ Motomura K, Oka T, Yokoyama M, Nakamura $\mathrm{H}$, Mori $\mathrm{H}$, Ode H, Hansman GS, Katayama K, Kanda T, Tanaka T, Takeda N, Sato H; Norovirus Surveillance Group of Japan: Identification of monomorphic and divergent haplotypes in the 2006-2007 norovirus GII/4 epidemic population by genome-wide tracing of evolutionary history. J Virol 2008;82: 11247-11262.
8 Kitahori Y, Inoue Y, Imanishi Y: Detection and genetic characterization of sporadic noroviruses in Nara Prefecture between April 2006 and September 2007. Jpn J Infect Dis 2008;61:89-90.

9 Kitahori Y, Inoue Y, Imanishi Y: Genetic characterization of sporadic noroviruses in Nara Prefecture: April 2007-March 2008. Jpn J Infect Dis 2009;62:82-83.

10 Kageyama T, Kojima S, Shinohara M, Uchida K, Fukushi S, Hoshino FB, Takeda N, Katayama K: Broadly reactive and highly sensitive assay for Norwalk-like viruses based on realtime quantitative reverse transcription-PCR. J Clin Microbiol 2003;41:1548-1557.

11 Kojima S, Kageyama T, Fukushi S, Hoshino FB, Shinohara M, Uchida K, Natori K, Takeda N, Katayama K: Genogroup-specific PCR primers for detection of Norwalk-like viruses. J Virol Methods 2002;100:107-114.

12 Iritani N, Kaida A, Kubo H, Abe N, Goto K, Ogura H, Seto Y: Molecular epidemiology of noroviruses detected in seasonal outbreaks of acute nonbacterial gastroenteritis in Osaka City, Japan, from 1996-1997 to 2008-2009. J Med Virol 2010;82:2097-2105.

13 Hoa Tran TN, Trainor E, Nakagomi T, Cunliffe NA, Nakagomi O: Molecular epidemiol ogy of noroviruses associated with acute sporadic gastroenteritis in children: global distribution of genogroups, genotypes and GII.4 variants. J Clin Virol 2013;56:185-193.

14 Iritani N, Seto Y, Kubo H, Murakami T, Haruki K, Ayata M, Ogura $\mathrm{H}$ : Prevalence of Norwalk-like virus infections in cases of viral gastroenteritis among children in Osaka City, Japan. J Clin Microbiol 2003;41:1756-1759.
15 Shinkawa N, Noda M, Yoshizumi S, Tokutake Y, Shiraishi T, Arita-Nishida T, Nishio O, Oka T, Hansman GS, Takeda N, Kimura H: Molecular epidemiology of noroviruses detected in food handler-associated outbreaks of gastroenteritis in Japan. Intervirology 2008;51: 422-426.

16 Chan-It W, Thongprachum A, Khamrin P, Kobayashi M, Okitsu S, Mizuguchi M, Ushijima H: Emergence of a new norovirus GII.6 variant in Japan, 2008-2009. J Med Virol 2012;84:1089-1096.

17 Iritani N, Kaida A, Abe N, Sekiguchi J, Kubo H, Takakura K, Goto K, Ogura H, Seto Y: Increase of GII.2 norovirus infections during the 2009-2010 season in Osaka City, Japan. J Med Virol 2012;84:517-525.

18 Lew JF, Valdesuso J, Vesikari T, Kapikian AZ, Jiang X, Estes MK, Green KY: Detection of Norwalk virus or Norwalk-like virus infections in Finnish infants and young children. J Infect Dis 1994;169:1364-1367.

19 Huhti L, Szakal ED, Puustinen L, Salminen M, Huhtala H, Valve O, Blazevic V, Vesikari T: Norovirus GII-4 causes a more severe gastroenteritis than other noroviruses in young children. J Infect Dis 2011;203:1442-1444.

20 Siebenga JJ, Vennema H, Zheng DP, Vinjé J, Lee BE, Pang XL, Ho EC, Lim W, Choudekar A, Broor S, Halperin T, Rasool NB, Hewitt J, Greening GE, Jin M, Duan ZJ, Lucero Y, O’Ryan M, Hoehne M, Schreier E, Ratcliff RM, White PA, Iritani N, Reuter G, Koopmans M: Norovirus illness is a global problem: emergence and spread of norovirus GII.4 variants, 2001-2007. J Infect Dis 2009;200: 802-812.

-21 Mathijs E, Denayer S, Palmeira L, Botteldoorn N, Scipioni A, Vanderplasschen A, Thiry E, Dierick K: Novel norovirus recombinants and of GII.4 sub-lineages associated with outbreaks between 2006 and 2010 in Belgium. Virol J 2011;8:310.
Sporadic Gastroenteritis in Nara

Prefecture, Japan 